ORIGINAL ResEARCH ARTICLE

\title{
Preparation and Research Progress of Polymer-based Flexible Conductive Composites
}

\author{
Xingqiang Chen,Suxing Fang,Zhenpin Hou \\ Materials and Chemical Engineering, Yangzhou University of Science and Technology, Jiangsu, China
}

\begin{abstract}
Abstract:The development of flexible wearable electronic devices is one of the future directions of technology development. Flexible conductive materials are important supporting materials for wearable electronic devices. Polymer has excellent flexibility, it is an important way to prepare flexible conductor from polymer-based conductive composites. In this paper, the research progress of polymer-based flexible conductive composites is summarized from the aspects of preparation and characterization methods. The key factors to realize flexible conductor are put forward, namely, the maintenance of excellent polymer elasticity and the realization of stable. The design and preparation of the extensible conductor with high elasticity matrix and nanofiller are introduced in detail, and the problems in the current research are summarized.
\end{abstract}

KEYWORDS: Flexible causes, polymers, conductive composite materials

Scientists predict that the next technological revolution in the world is the development and application of wearable equipment. The flexible conductor is an important base material for wearable equipment. Flexible Conductors (Flexible Conductors) refers to the material in a large strain. It can maintain good electrical performance of new intelligent electronic materials for electronic devices [1], electronic skin [2], intelligent fabrics [2], Intelligent robotic arm [3], deformable electronic components $[3,4]$ and other new intelligent materials that has very important application.

The development of polymer-based conductive composites provides a new idea for the preparation of stretchable conductors. In polymer-based stretchable conductive composites, the polymer provides excellent high elasticity, and the conductive filler forms a good conductive network in the polymer matrix. The 2 factors have to be satisfied in order to maintain good electrical performance: 1) to maintain high elasticity of the polymer; 2) to have a stable or deformable conductive network. A wide range of in-depth studies have been carried out for the preparation of polymer-based tensile conductive composites, and the domestic and foreign scholars have conducted extensive and in-depth studies. This paper will summarize the representative methods of preparation and research.

\section{Preparation Method}

\subsection{High elasticity matrix and nano filler}

The first method used is to select the polymer with excellent elasticity and high aspect ratio; high conductivity of the nano filler, and improve the content of the filler. This method is mainly used to form a stretchable conductive network by using intertwined entanglement between high content and high aspect ratio nanostructured fillers in an elastic matrix. The matrix is similar to the random coil structure of the polymer material. (EPMS), polyurethane (TPU), styrene- (ethylene-butadiene copolymer) -styrene block copolymer (SEBS) and etc. Fillers include carbon Nanotubes (CNTs), nano-silver (AgNWs), graphene, expanded graphite and so on. Among them, CNTs are the most widely used due to the high aspect ratio, intrinsic flexibility and relatively mature preparation process. Japan's University of Tokyo Sekitani et al. [4] are the first to carry out research. They use the direct blending way. Firstly, they used super long single-walled carbon nanotubes (SWCNTs, length $1 \mathrm{~mm}$ above) and ionic liquid mixture to form bulky gel (bulky gel). Then, it is blended with PDMS to form a stretchable conductor. Due to the strong force between the long SWCNTs and the tangential effect of the SWCNTs, the conductivity of the composites with the weight of $20 \% 57 \mathrm{~S} / \mathrm{cm}$; and the

Copyright (C) 2017 -. This is an Open Access article distributed under the terms of the Creative Commons Attribution-NonCommercial 4.0 International License (http://creativecommons.org/licenses/by-nc/4.0/), permitting all non-commercial use, distribution, and reproduction in any medium, provided the original work is properly cited. 
electronic devices prepared based on the above flexible conductor materials still maintain excellent mechanical and electrical properties when they are $70 \%$ deformed in both directions. In order to improve the dispersibility of CNTs and improve the conductivity of composites, the conductivity of SEBS/CNTs $(20 \%)$ composites was improved when the mass fraction of CNTs was increased to $20 \%$ by high-speed mixing method. The conductivity of the composite had decreased rapidly with the tensile strain. When the strain reaches $150 \%$, the conductivity decreases by 3.5 orders of magnitude. In order to further improve, the composite $(20 \%)$ was prepared into a solution and spin-coated on a pure SEBS surface to form a highly elastic conductive composite, in which the pure SEBS provided high elasticity and large deformation. This resulting conductivity of the composite material reduced by only 0.5 orders of magnitude at a strain of $150 \%$. In addition, AgNWs are widely used to prepare flexible composites due to their high conductivity and good flexibility. North Carolina State University Song et al. [7] placed AgNWs with a diameter of $90 \mathrm{~nm}$ and a length of about $6 \mu \mathrm{m}$ in a flexible PDMS in a screen-print manner. The reverse of the flexible antenna can be used for wireless transmission and so on.

\subsection{Preparation of Structural Drawn Conductors}

In the method 1.1, most of the material can be stretched from the point of view. More research is focus on the conductive network structure to form a multi-level folding of the stretchable conductive network structure, and enhance its structural extensibility of the method. The latter also known as the structure of stretchable conductor. The current application of this method is common, especially the following method:

(1) Pre-fabricating a highly conductive material into a wavy or multi-layered folded structure and then recombining with a flexible substrate. North American University Fan et al [8] used the electron beam evaporation and chemical etching method to prepare the metal conductor into a multi-level wave type, Piano curve type, Cock curve and other conductive structure with a folding structure (The structure is shown in Fig.1). Then, it is combined with the prestrained PDMS to obtain a conductor with a strain greater than $100 \%$. It is pointed out that the size of the material will significantly affect the stretch ability of the flexible conductor. The Shin et al. [9] of the University of Texas at Dallas [9] prepared CNT forms on silicon wafers using a vapor deposition process catalyzed by Fe, and then, by pouring PU solution penetrates into CNTs. CNTs are intertwined in CNTs, the formation of similarity to accordion-type conductive network makes the fracture elongation of the composite (CNTs only $0.1 \%$ ) reach $1400 \%$. In $20 \%$ and $40 \%$ of the tensile strain has good conductivity retention and bending. Twisting can still maintain the original conductivity.

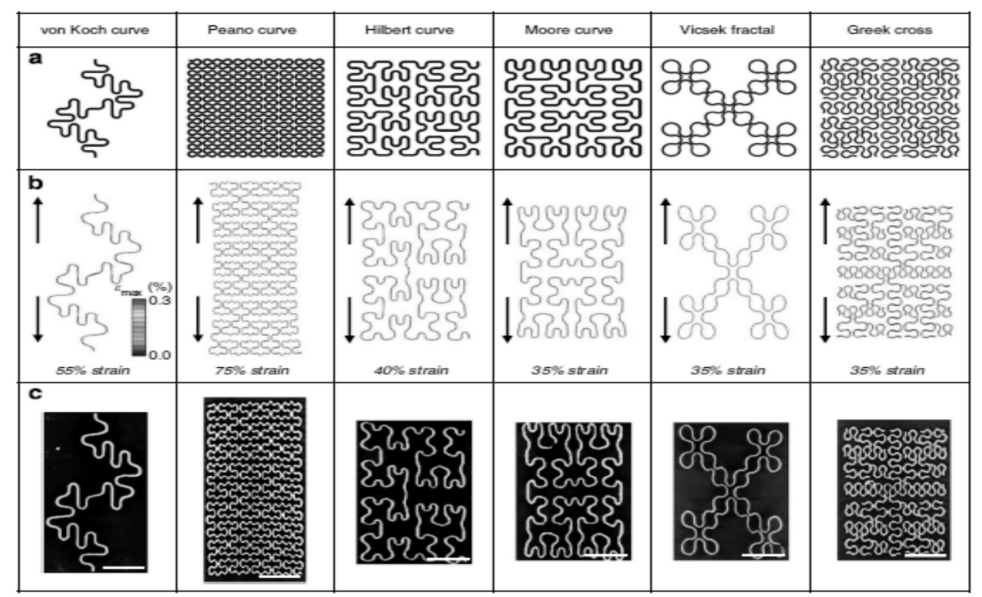

Fig.1

(2) Placing the highly conductive material on the pre-stretched highly elastic substrate, the conductor will forms a wavy or folded conductive structure upon recovery of the substrate. Wang et al. [10] of the Hong Kong Polytechnic University employed a layer of copper with a thickness of 100 to $300 \mathrm{~nm}$ on the surface of the pre-stretched PDMS, by using a polyelectrolyte through a metal electroless deposition. In the process of matrix retraction of the nanocrystalline copper layer formed a wave structure. The successful preparation of the stretchable conductor shows in Fig. 2 for its mechanism diagram. Zhu et al. [11] also used the wave-shaped conductive network generated during the pre-stretching and retraction of the substrate to fabricate a flexible conductor that penetrates the liquid silicone rubber onto the surface with PDMS as the substrate. The conductivity of the composite material is kept within the range of less than $50 \%$ of the strain after 5 times of stretching (when the strain is 50\%). The reason is after repeated stretching, the composite conductive material is formed in the AgNWs. In the process of relaxation, the PDMS / AgNWs on the upper layer of the substrate form a folded wave-like conductive structure. 


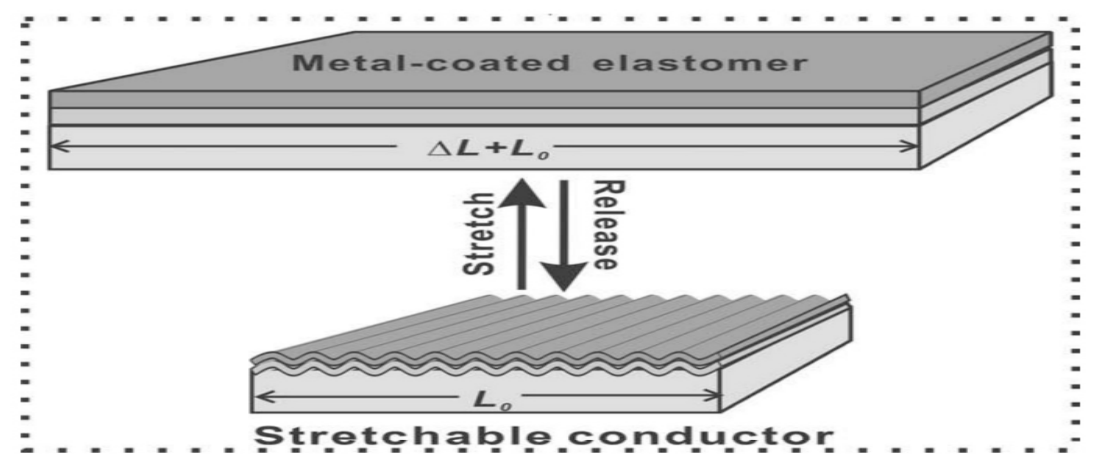

Fig. 2

(3) By using morphological control method to increase the contact between the filler. It can be stretched by enhance the conductivity of the network. Zhang et al. [12] of the Los Alamos National Laboratory of the United States extracted the CNTs band (CNTR) from the spinneretable CNTs and held them in the PDMS. It subjected to stretching and relaxation cycles to form a tensile structure Network. Due to CNTR has a good orientation structure, and good contact in the process of stretching between the CNTs only axial slip. So, the conductivity of the composite tensile strain reached $100 \%$ when the conductivity remains. The study shows that the orientation of CNTs is beneficial to maintain the conductivity of the composites during stretching. Unlike the Jia task group, North Carolina State University, et al. [13] which directly placed the CNTR on the PDMS substrate surface, and co-stretched CNTR/PDMS and relaxed. By carefully regulating the CNTs and PDMS interface. The control of CNTs and PDMS in the relaxation process of the degree of recovery, so that CNTs occur between the bending and bending and spontaneously buckle the formation of folded conductive network. When the strain is $100 \%$ stretch and relaxation, the composite material conductivity in the re-stretch remains unchanged. The mechanism diagram showed in Fig. 3. In the PU/CNTs composites, the CNTs are oriented in the tensile direction by the pre-straining of the TPU/CNTs composites prepared by the fusion method. Then the thermal annealing treatment is carried out such in the orientation of the CNTs. While increasing the local contact and mutual buckle, the mechanism diagram as shown in Fig. 4 . When the CNTs mass fraction is $30 \%$, the conductivity of the composite remains constant and the tensile strain reaches $300 \%$.

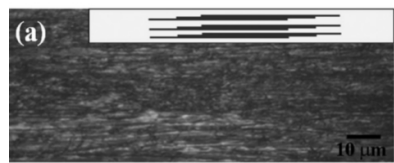

(b)
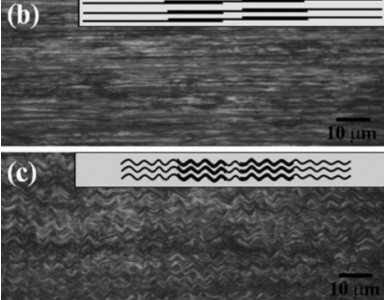

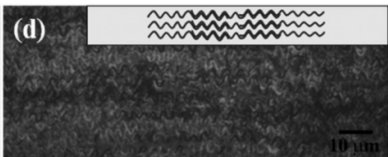

(e)

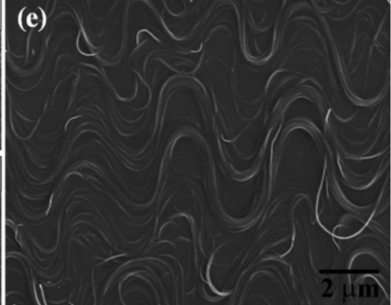

Fig. 3.

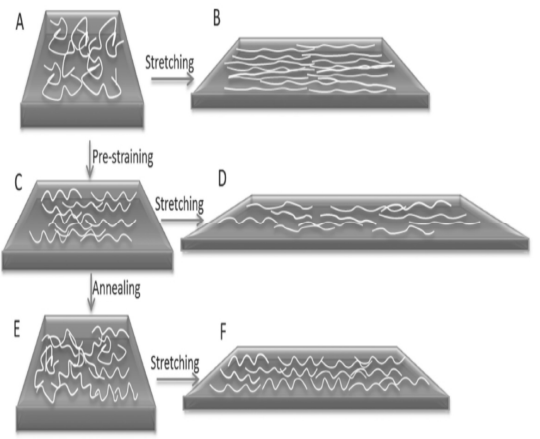

Fig. 4

(4) Through the design of flexible graphene or carbon nanotubes of airgel. The aerogels as a conductor back to fill the preparation of conductive composite materials. Carnegie Mellon University Kim et al. [15] prepared a composite of the SWCNTs by filling the SWCNTs airgel with PDMS, and the composites were prepared when the tensile strain 
reached $100 \%$. The conductivity remains the same. Dalian University of Technology Qiu Qishan Project Group [16,17] also prepared a flexible compressible conductive composite by refilling PDMS into graphene airgel.

The method of forming a folded network structure in the matrix is one of the most widely used methods for the preparation of flexible conductors. It is found that the orientation structure of the filler is beneficial to the preparation of the stretchable conductor.

\subsection{Ionic liquid assistance or doping}

The ionic liquid is assisted or doped which enhances the force between the fillers and provides a variety of conductive modes. In the SWCNTs/elastic matrix composites, the ionic liquids with reactive activity were added to the SWCNTs/elastic matrix composites, which promoted the SWCNTs dispersion and also formed a strong effect between the SWCNTs. So, the composites could withstand the large strain Electrical performance. Korea Institute of Science and Technology Kim et al. [19] sprayed CNTs with ionic liquid gels and silicone rubbers on elastomeric substrates, then doping in nitric acid vapor. The results show that after doping of the composite material in the twentieth stretch, the conductivity of $100 \%$ deformation only by the initial $19 \mathrm{~S} / \mathrm{cm}$ reduced to $18 \mathrm{~S} / \mathrm{cm}$. Hong Kong Polytechnic University Shang Chungmin Task Group [20,21] CNTs were uniformly dispersed into the PU matrix by means of ionic liquids. Then the composite material for removing the ionic liquid was thermally stretched 10 times at $100^{\circ} \mathrm{C}$ to make CNTs in the matrix. When the mass fraction of CNTs is $10 \%$, the conductivity of the composites increases slightly. When the tensile deformation reaches $100 \%$ and can maintain several cycles.

\subsection{Fibrous conductive polymer based flexible composites}

In the fibrous conductive polymer-based flexible composite material, flexible conductors are prepared by the flexibility and weaving of fibers. The preparation methods include conventional spinning and electrospinning. Daniel et al. [22] of the University of Texas at Dallas [22] wound flexible CNTs on the surface of the stretched rubber fibers, followed by CNTs forming a multilayer stretchable structure during the relaxation of the rubber fibers. Then, it obtains a stretchable conductor with multi-layer structure with fiber as core and CNTs as shell. The rubber layer can be further coated on the CNTs layer to form a multilayer structure conductor. The results show that the core- $1000 \%$ tensile strain when the resistivity change is less than 5\%, reflecting the excellent stretchability, its structure as shown in Fig. 5. Liu et al. [23] of Duke University of the United States used a method of applying a layer of CNTs to the surface of polyisophthalamide (PMIA) fibers during the formation of poly. The orientation of CNTs was promoted during the stretching process, and the obtained composite fibers were maintained at $200 \%$ tensile strain. Ma et al [24] of Sungkyunkwan University [24] used the petal-like nano silver directly and PU compound and spinning due to the petallike nano-silver and PU between the strong, composite fiber conductivity up to $41245 \mathrm{~S} / \mathrm{cm}$, and Elongation at break up to $776 \%$. Then the composite fibers were prepared into weft knitted fabrics, which had a conductivity change of less than $5 \%$ when the tensile strain reached $200 \%$.
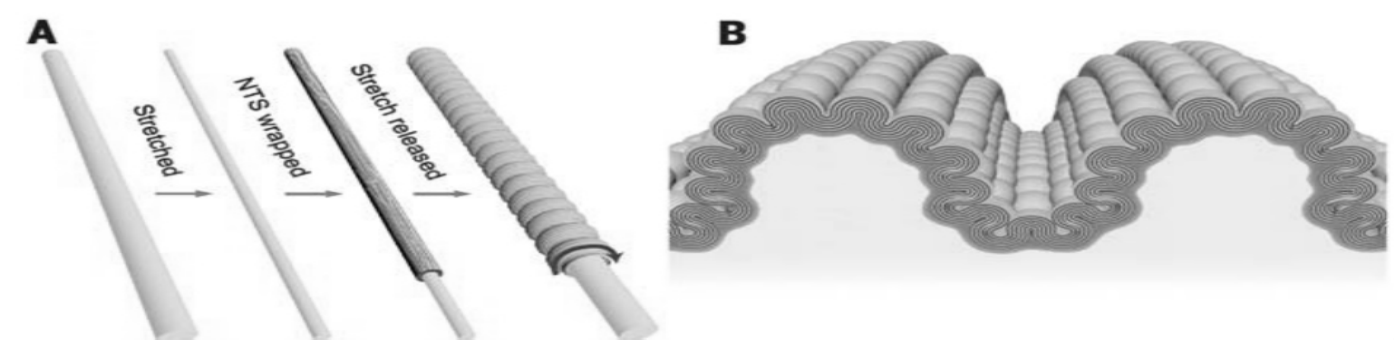

Fig. 5

Electrostatic spinning is an effective method for the preparation of micro-nano and flexible fibers. Persa et al. [25] of the University of Illinois at Urbana-Champaign of the United States [25] changed the spinning collection method by using a roller-type ordered polyvinylidene fluoride-trifluoroethylene copolymer (P (VDF-TrFE)) of the electrospinning, and loaded on the PDMS surface to achieve low pressure under the effect of strain induction. Based on the basic method of high-voltage electrospinning, a new type of spinning nozzle reciprocating spinning device can be used to obtain a distorted structure of polyvinylpyrrolidone/polyethylene dioxythiophene: poly Styrene sulfonate (PVP/PESOT: PSS) micro-nanofibers. Since the fibers are stored in a twisted structure, they can be used for flexible stretchable conductors.

\subsection{Other methods}

In addition to the above three categories, there are many outstanding results, such as the natural rubber after the swelling and diffusion of graphene [27] and the preparation of flexible conductive hydrogels [28]. British University 
of Surrey Boland et al. [27] used vulcanized rubber to swell and dissolve the vulcanized natural rubber with suitable solvents, and to penetrate into the graphene by diffusion. The results show that the graphene is gradually infiltrated from the surface of the natural rubber, and the permeability of the graphene is increasing with the infiltration time. The higher the conductivity of the composite material, the higher the conductivity of the composite material is $0.5 \%$ (volume fraction). After infiltration for 50 hours, and the percolation value of the composite material is only $0.12 \%$ (volume fraction). For its flexible test, the elongation of the material is greater than $800 \%$. The composite material with low graphene mass fraction $(0.2 \%)$ is an ultra-sensitive strain sensor with a GF (gauge factors) of 35. Ding et al., Carnegie Mellon University, USA [28] prepared polyaniline materials with micro-nanostructures by photo-curing heparin-acrylate as a scaffold for the design of dual-network hydrogels. It was found that the composite hydrogel had a storage modulus of only $(900 \pm 100) \mathrm{Pa}$ and a resistance value of $4.17 \Omega(1 \mathrm{kHz})$. It was a flexible conductive hydrogel with excellent properties and a wide range of artificial skin application. The preparation method provides the ideas and experiences for the design and preparation of polymer-based stretchable flexible conductors from the aspects of substrate and filler selection, structural design of conductive network and preparation method. The main factors influencing the extensibility of the composites are the degree of contact between the fillers, the structure of the conductive network and the high elasticity of the composites. However, there are some problems in the above studies, including: 1) higher filler content: when using direct composite, the use of filler is too high, such as CNTs mass fraction of up to $10 \%$ or even 30\%; far more than CNTs percolation, which will damage the high elasticity of composite materials and cannot reflect the advantages of nano-filler. Increasing the filler content ensures sufficient contact between the fillers to improve the conductivity of the composite material, but it will reduce the high elasticity. 2) The extensibility of the conductive network structure is low. When the polymer-based tensile conductor is prepared by the direct recombination method, CNTs are uniformly dispersed in the matrix and CNTs are crimped. A certain tangent but weak interaction force, the structure of the conductive network is also very low stretchability. This will seriously affect the stretchability of composite materials, resulting in the conductivity of the composite material under great strain attenuation too fast Poor circulation. 3) In the preparation of a stretchable conductor with a folded conductive network, the post-processing is complicated and has a difficult handling. In the repeated tension relaxation, it is difficult to accurately predict the appropriate tensile strain and the number of cycles, which requires a large number of experimental attempts. In the regulation of the interfacial force between CNTs and the substrate, the interfacial force between the test and the theoretical simulation large difficulty. So, the theory is difficult to achieve prediction, but also need to try to experiment.

\section{Research Characterization}

In the study of flexible conductors, the most common concern is the conductivity of the conductor, the flexibility or elongation of the composite, the change in conductivity and stability of the conductor under various deformations. The key factors that determine the performance of these properties are the structure of the conductive network and the content of the filler. The characterization of these two factors mainly includes intuitive morphology and indirect structure characterization.

\subsection{Flexible Characterization}

The method used in characterize the performance of flexible conductors is to characterize the tensile modulus or elongation at break of the conductor $[4,28,29]$. Then, test the conductor in the tensile $[6,12]$, bend $[9,20][9,20]$ and other changes in the case of conductivity changes and repeatability. Once again through the conductor to connect electronic devices such as LED lights [11], characterization of electronic device performance changes.

The first thing used to characterize the material is its modulus. Kujawski et al. [29] found that the elastic modulus was only $1.44 \mathrm{MPa}$ when the filling amount reached 25\% (mass fraction) after padding with PDMS (PDMS and curing agent ratio of 20: 1). Good flexibility, indicating that it can be used for flexible electrode materials. Ding et al. [28] found that the prepared double-network hydrogel had a storage modulus of only $(900 \pm 100) \mathrm{Pa}$, which was lower than that of the more flexible materials $(1 \sim 10 \mathrm{kPa})$.

Followed by the test conductor in a variety of strain conditions, it changes in conductivity. This method uses the most and is the most intuitive of a characterization method. Sekitani et al. [4] first compared the SWCNTs film and its composite materials and conventional commercial conductive rubber. The results show that the conductivity of the SWCNTs film is $40 \%$ of the tensile strain using the conventional conductive rubber. The SWCNTs composite material can be repeated more than 103 times when the strain is 50\% in the cyclic repeatability. Zhang et al. [12] designed the preparation of the synchronous test device to characterize the conductor in the process of stretching and circulation of the conductivity changes in the law, found through the CNTs prepared by the flexible conductor in the elongation of $120 \%$ to remain unchanged. The number of cycles is up to 30 times. Shin et al. [9] tested the change in conductivity during bending and torsion in order to demonstrate the properties of flexible conductors prepared by CNTs, and found that the conductivity of the composites varied by only $1 \%$ during the bending process in the conductivity change of less than 4\%; and can maintain more than 100 times the bending or folding. (HTVSR) / CNTs composites with chitosan pretreated CNTs with the change of bending or torsional angle, the results show that when the CNTs content is low, the 
conductivity rate increases with the increase of the bending or twisting angle; and the conductivity does not change with the bending or twisting angle when the CNTs content is high, which shows that it has good flexibility. The use of flexible conductors to connect electronic devices such as LED, LED lights with the flexibility of the degree of deformation of the conductor to prove the degree of brightness. Xu et al. [11] used this method to connect the AgNWs/PDMS wires to the LEDs and energize them to find that the brightness of the LEDs was reduced at the time of stretching. The brightness of the LEDs was unchanged after the conductor was folded, and the AgNWs/PDMS has good flexibility.

\subsection{Characterization of the form}

Morphological representations can visually observe the structure of conductive networks, including various microscopic techniques such as SEM, TEM, and so on. For example, Zhu et al. [13] and Lin et al. [14] used SEM to characterize the structural changes of the composites before and during the pre-strain process. The results of Zhu et al. [13] show that CNTs undergo a process of stretching and recovering at the same time during the stretching process. After several stretching, the CNTs form a wavy conductive network, which is conducive to the use of composite materials in the process does not change with the strain changes. Lin et al. [14] have shown that the conductive network of CNTs undergoes a process such as tensile orientation, solution orientation and local lap during the pre-strain process of TPU/CNTs composites, resulting in a stable lap between CNTs. The internet structure can maintain the stability of the conductivity.

For CNTs, CNTs aerogels, graphene aerogels, SEM or TEM visual morphology is the most commonly used. Zhang et al. [12] demonstrated that CNTs have obvious entanglement in CNTs by SEM and TEM, which is helpful to maintain the stability of the conductivity of the composites during stretching. Qiu Jie Shan, etc. through the SEM proved that the preparation of graphene aerogels have obvious hollow and have a strong role between each other, which ensures the airgel in the strain when the strain stability.

\subsection{Characterization of structures}

The morphological representation of the conductive network is limited to the size factor. Only the smaller range can be observed, and the representation of the structure is helpful to further prove the morphological changes, which is statistically significant. Fu Qiang et al. [14] In order to prove the process of orientation, solution orientation and local re-lap of CNTs in TPE / CNTs composites during pre-strain and heat treatment, one-dimensional and two-dimensional X-ray diffraction and Raman spectra. It is proved that the CNTs are oriented in the pre-strain process, and then the CNTs are decoupled during the annealing process. Finally, the conductive network is formed by local overlap and intertwined to ensure that the composite material is in the process of stretching in the conductivity of the same.

\section{Summary}

Flexible conductive is an important supporting material for wearable equipment. Its preparation and research is of great significance. The use of highly flexible and highly flexible polymeric materials allows the preparation of highperformance polymer-based flexible conductors. However, how to use a controlled and predictable experimental to form a folded structure in less conductive fillers is to produce high-rate polymer. The key to stretch the conductor are also the current focus of attention.

\section{References:}

1. Sekitani T, Yokota T, Zschieschang U, et al. Organic nonvolatile Memory transistors for flexible sensor arrays [J]. Science, 2009, 326: 1516-1519.

2. Benight S J, Wang C, Tok J B H, et al. Stretchable and selfhealing Polymers and devices for electronic skin [J]. Prog. Polym. Sci. , 2013, 38 (12): 1961-1977.

3. Rogers J A, Someya T, Huang Y. Materials and mechanics for Stretchable electronics [J]. Science, 2010, 327: $1603-1607$.

4. Sekitani T, Noguchi Y, Hata K, et al. A rubberlike stretchable Active matrix using popularity around [J]. Science, 2008,321:1468-1472.

5. Li Y, Shimizu H. Toward a stretchable, elastic, and electrically Conductive nanocomposite: morphology and properties of poly [Styrene-b- (ethylene-co-butylene) - B-styrene / multiwalled carbon Nanotube composites fabricated by high-shear processing [J].Macromolecules, 2009, 42: 2587-2593.

6. Li Y, Zhao L, Shimizu H. Electrically conductive Materials with high stretchability and excellent elasticity by a surface Coating method [J]. Macromol. Rapid Commun , 2011,32:289-294.

7. Song L, Myers A C, Adams J J, et al. Stretchable and reversibly Deformable radio frequency eth based on silver nanowires[J]. ACS Appl. Mater. Interfaces, 2014, 6: 4248-4253.

8. Fan J A, Yeo W H, Su Y, et al. Fractal design concepts for Stretchable electronics [J]. Nat. Commun , 2014, 5: 163-180.

9. Shin M K, Oh J, Lima M, et al. Elastomeric conductive composites Based on carbon nanotube forests [J]. Adv. Mater. , 2010,22: 2663-2667. 
10. Wang $\mathrm{X}, \mathrm{Hu} \mathrm{H}$, Shen $\mathrm{Y}$, et al. Stretchable Ultrahigh tensile strain and stable metallic conductance enabled by prestrained polyelectrolyte nanoplatforms [J]. Adv. Mater. ,2011,23: 3090-3094.

11. Xu F, Zhu Y. Highly conductive and stretchable silver nanowire[J]. Adv. Mater., 2012, 24: 5117-5122.

12. Zhang Y, Sheehan C J, Zhai J, et al. Polymer-embedded carbon Nanotube ribbons for stretchable donor [J]. Adv. Mater. ,2010, 22: 3027-3031.

13. Zhu Y, Xu F. Buckling of aligned carbon nanotubes as stretchable A new manufacturing strategy [J]. Adv. Mater. , 2012,24: 1073-1077.

14. Lin L, Liu S, Fu S, et al. Fabrication of highly stretchable Advertising via morphological control of carbon nanotube network[J]. Small, 2013, 9: 3620-3629.

15. Kim K H, Vural M, Islam M F. Single-walled carbon nanotube Aerogel-based public around [J]. Adv. Mater. , 2011,23:28652869.

16. $\mathrm{Hu} \mathrm{H,} \mathrm{Zhao} \mathrm{Z,} \mathrm{Wan} \mathrm{W,} \mathrm{et} \mathrm{al.} \mathrm{Ultralight} \mathrm{and} \mathrm{highly} \mathrm{compressible} \mathrm{Graphene} \mathrm{aerogels} \mathrm{[J].} \mathrm{Adv.} \mathrm{Mater.} \mathrm{,} \mathrm{2013,} \mathrm{25:} 2219-2223$.

17. Hu H, Zhao Z, Wan W, et al. Polymer / graphene hybrid aerogel With high compressibility, conductivity, and 'sticky' superhydrophobicity[J]. ACS Appl. Mater. Interfaces, 2014, 6:

18. 3242-3249.

19. Zhao L, Li Y, Qiu J, et al. Reactive bonding mediated high Mass loading of individualized single-walled carbon nanotubes in An elastomeric polymer [J]. Nanoscale, 2012, 4: 6613-6621.

20. Kim T A, Kim H S, Lee S S, et al. Single-walled carbon Nanotube / silicone rubber composites for compliant electrodes [J]. Carbon, 2012,50: 444-449.

21. Shang S, Gan L, Yuen M C W, et al. Carbon nanotubes based High temperature vulcanized silicone rubber nanocomposite with Excellent elasticity and electrical properties [J]. Composites Part A, 2014, 66: 135-141.

22. Shang S, Zeng W, Tao X M. High stretchable MWNTs / Urethane guided nanocomposites [J]. J. Mater. Chem., 2011, 21: 72747280.

23. Liu Z F, Fang S, Moura F A, et al. Hierarchically buckled Sheath-core fibers for superelastic electronics, sensors, and Muscle [J]. Science, 2015, 349: 400-404.

24. Jiang S, Zhang H, Song S, et al. Highly stretchable Fiber from few-walled carbon nanotubes coated on poly (m-phenylene Isophthalamide) polymer core / shell structures [J]. ACS Nano, 2015, 9: 10252-10257.

25. Ma R, Kang B, Cho S, et al. Extraordinarily high conductivity Of stretchable fibers of polyurethane and silver nanoflowers [J]. ACS Nano, 2015, 9: 10876-10886.

26. Persano L, Dagdeviren C, Su Y, et al. High performance Piezoelectric devices based on aligned arrays of nanofibers of poly (Vinylidenefluoride-co-trifluoroethylene) [J]. Nat. Commun , 2013,4: 67-88.

27. Sun B, Long Y Z, Liu S L, et al. Fabrication of curled conduc-ting polymer microfibrous arrays via a novel electrospinning Method for stretchable strain sensors [J]. Nanoscale, 2013,5:7041-7045.

28. Boland C S, Khan U, Backes C, et al. Sensitive, high-strain, High-rate bodily motion sensors based on graphene- rubber Composites [J]. ACS Nano, 2014, 8: 8819-8830.

29. Ding H, Zhong M, Kim Y J, et al. Biologically derived soft Conducting hydrogels using heparin-doped polymer networks[J]. ACS Nano, 2014, 8: 4348-4357.

30. Kujawski M, Pearse J D, Smela E. Elastomers filled with Exfoliated graphite as compliant electrodes [J]. Carbon, 2010,48: 2409-2417. 